\title{
NEUTRON-SCATTERING EXPERIMENTS ON THE TWO-DIMENSIONAL EASY-PLANE ANTIFERROMAGNET $\mathrm{K}_{2} \mathrm{FeF}_{4}$
}

\author{
M. P. H. THURLINGS*, E. FRIKKEE ${ }^{\dagger}$ and H. W. DE WIJN* \\ *Fysisch Laboratorium, Rijksuniversiteit, P.O. Box 80.000, 3508 TA Utrecht, The Netherlands \\ ${ }^{\dagger}$ Netherlands Energy Research Foundation ECN, 1755 ZG Petten (N.H.), The Netherlands
}

\begin{abstract}
Spin-wave dispersion and sublattice magnetization of $\mathrm{K}_{2} \mathrm{FeF}_{4}$ have been examined by neutron scattering and analyzed in terms of renormalized spin-wave theory appropriate for the easy-plane square lattice. Numerical results are $J=(-11.5 \pm 0.6) \mathrm{cm}^{-1}$, $D=(3.6 \pm 0.5) \mathrm{cm}^{-1}$, and $E(T=0)=(-0.37 \pm 0.10) \mathrm{cm}^{-1}$. Near $T_{\mathrm{N}}$ the sublattice magnetization follows a power law, with $T_{\mathrm{N}}=(62.7 \pm 0.6) \mathrm{K}$ and $\beta=0.13 \pm 0.01$.
\end{abstract}

The main objective of the present investigation is to determine the spin-wave dispersion and sublattice magnetization in the two-dimensional (2D) easy-plane antiferromagnet $\mathrm{K}_{2} \mathrm{FeF}_{4}$. Accordingly, we performed both elastic and inelastic neutron scattering in the temperature range $4.2-72 \mathrm{~K}$ with a triple-axis spectrometer at the HFR reactor in Petten. In fig. 1 the results are collected of a number of constant- $Q$ scans along the [100] direction in the magnetic Brillouin zone. Two distinct magnon branches could be tracked up to $\zeta_{x}=$ $a k_{x} / 2 \pi \approx 0.2$. Further experiments showed the energy of both branches to be independent of $k_{z}$, i.e. the spin waves are truly $2 \mathrm{D}$.

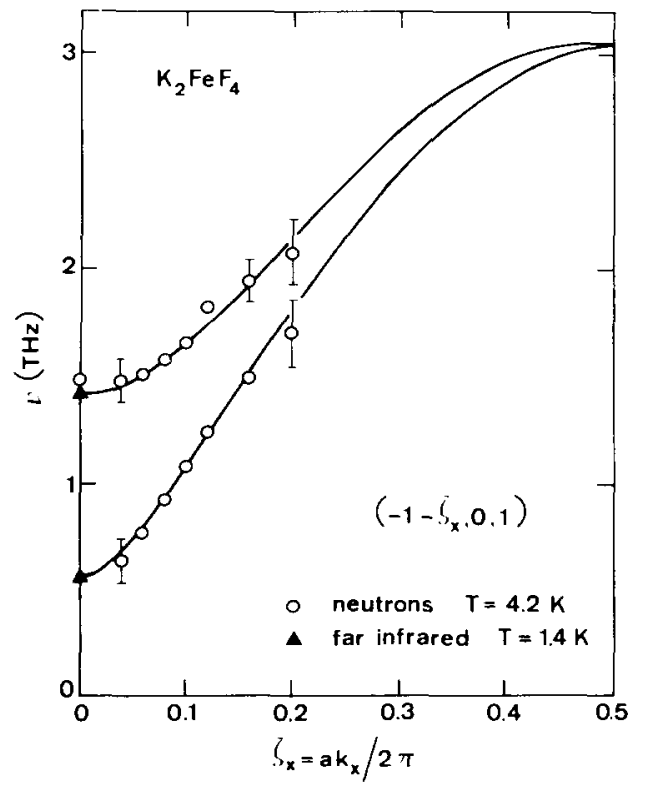

Fig. 1. Spin-wave dispersion at $4.2 \mathrm{~K}$ versus $k_{x}$, with $k_{y}=0$. The solid curve represents spin-wave theory. The black triangles indicate supplementary results of far-infrared spectroscopy.
To calculate the spin-wave energies we assume the Hamiltonian

$$
\mathcal{K}=|J| \sum_{\langle i, j\rangle} S_{\mathrm{i}} \cdot S_{\mathrm{j}}+\sum_{i}\left[D S_{\mathrm{i}}^{z 2}+E\left(S_{i}^{x 2}-S_{i}^{y 2}\right)\right]
$$

in the usual notation; $D(>0)$ represents the outof-plane anisotropy and $E$ the smaller in-plane anisotropy. Holstein-Primakoff transformation of eq. (1), followed by the introduction of spin waves and diagonalization, leads to two spin-wave branches, whose energies are to lowest order in $1 / 2 S$ given by

$$
\begin{aligned}
h \nu_{ \pm}(\boldsymbol{k})= & 4|J| S\left\{[1+(D-3 E) / 4|J|]^{2}\right. \\
& \left.-\left[\gamma_{k} \pm(D+E) / 4|J|\right]^{2}\right\}^{1 / 2} .
\end{aligned}
$$

Here, $\gamma_{k}=\cos \frac{1}{2} k_{x} a \cos \frac{1}{2} k_{y} a$. The $\nu_{+}$modes, having the lowest energy, correspond to mainly in-plane spin deviations; the $\nu_{-}$modes to mainly out-of-plane deviations. A best fit is made of eq. (2) to the data, with the results $J=(-11.7 \pm 0.6)$ $\mathrm{cm}^{-1}, D=(3.0 \pm 0.5) \mathrm{cm}^{-1}$, and $E=(-0.20 \pm$ $0.05) \mathrm{cm}^{-1}$. It is represented by the solid curves in fig. 1, which are in excellent agreement with experiments. In a more precise calculation, eq. (2) is extended to include corrections up to first order in $1 / 2 S$. The first-order renormalized eq. (2) yields a dispersion which only marginally deviates from the solid curves, but the spin-wave parameters are modified to $J=(-11.5 \pm 0.10) \mathrm{cm}^{-1}, D=(3.6 \pm$ $0.5) \mathrm{cm}^{-1}$, and $E=(-0.37 \pm 0.10) \mathrm{cm}^{-1}$.

To determine the variation with temperature of the sublattice magnetization $M$ the intensity of the magnetic (100) reflection was recorded (fig. 2). In the low-temperature regime, $M(T)$ has also been 
calculated from the above spin-wave formalism including temperature-dependent renormalization (solid curve). Good agreement is obtained up to $\sim 50 \mathrm{~K}$. Here, the result of $J, D$, and $E$ just obtained have been inserted. It has been assumed that $J$ and $D$ are constant, and $E$ scales with the asymmetry parameter of the field gradient derived from Mössbauer experiments [1]. These assumptions also lead to a correct description of the observed temperature variation of the spin-wave energies. For comparison, in fig. 2 also the result is given, as the dashed line, of the same calculation but with both $D$ and $E$ taken independent of temperature. To demonstrate the improvement obtained by renormalization, the result of an unrenormalized calculation is finally given as the dasheddotted line, which departs from experiment beyond $\sim 30 \mathrm{~K}$.

In the critical regime the sublattice magnetization displays a well-defined transition, going to zero at $T_{\mathrm{N}}=(62.7 \pm 0.6) \mathrm{K}$ according to a single power law with exponent $\beta=0.13 \pm 0.01$. The value for $\beta$ is to be compared with Onsager's result $\beta=\frac{1}{8}$ for the $2 \mathrm{D}$ Ising system. It should be noted that $T_{\mathrm{N}}$ is confirmed by critical-scattering experiments not discussed here. The results in the critical regime, however, deviate from those of the Mössbauer work [2], where a large transition region from 63 to $70 \mathrm{~K}$ was found. In $\mathrm{Rb}_{2} \mathrm{FeF}_{4}$, Wertheim et al. [3] have reported similar effects. Possibly, residual short-range order within the $a-b$ planes still shows up in the Mössbauer spectra somewhat above $T_{\mathrm{N}}$. As a matter of fact, $2 \mathrm{D}$ critical scatter-

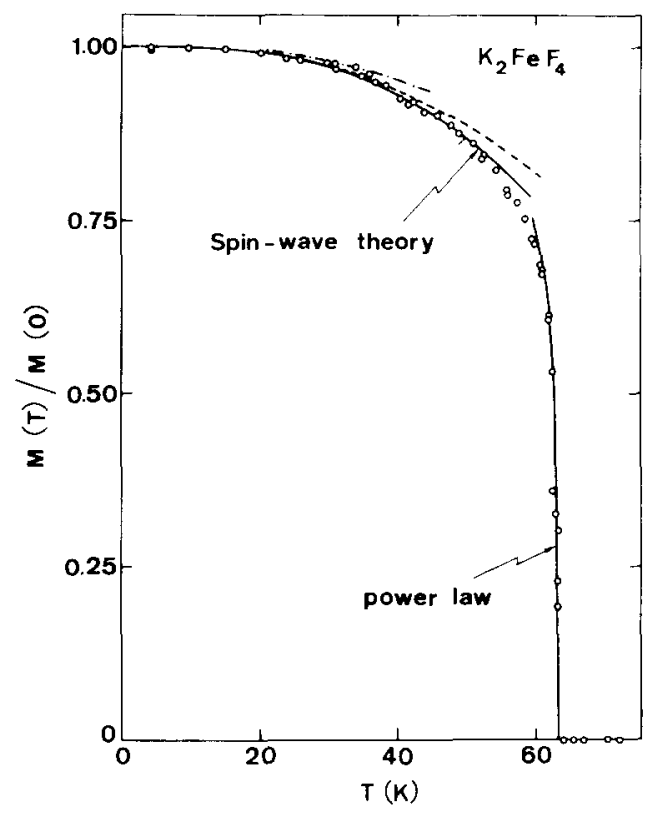

Fig. 2. Normalized sublattice magnetization versus temperature.

ing, which essentially reflects short-range order, is observed to persist in the same temperature regime.

\section{References}

[1] M. P. H. Thurlings, A. M. van Diepen and H. W. de Wijn, this conference, ch. 12, paper $5 \mathrm{Z5}$.

[2] M. P. H. Thurlings, E. M. Hendriks and H. W. de Wijn, Solid State Commun. 27 (1978) 1289.

[3] G. K. Wertheim, H. J. Guggenheim, H. J. Levinstein, D. N. E. Buchanan and R. C. Sherwood, Phys. Rev. 173 (1968) 614. 